$\mathrm{AB} 0831$

THE INFLUENCE OF REMISIVE AND ANTIINFLAMMATORY TREATMENT ON AXIAL MOBILITY IN PATIENTS WITH ANKYLOSING SPONDYLOARTRITIS

E. Russu, L. Chislari, R. Larisa, V. Cazac. Rheumatology and Nephrology, SMPU "Nicolae Testemitanu", Chisinau, Moldova, Republic of

Background: In advanced ankylosing spondyloarthritis (AS), bone ankylosis or ossification of the involved joints can make the chest practically immobile, decrease its compliance, or even lead to intercostal muscle atrophy.

Objectives: The purpose of the study was to evaluate chest involvement in AS by measuring toracoabdominal movements during quiet breathing, by dividing the chest and abdominal contribution to the current volume, by inductive plethysmography methods.

Methods: 60 consecutive patients were recruited from the Rheumatology Department of the Republican Clinical Hospital. They were selected based on AS diagnosis, with no existing cardiovascular or neuromuscular diseases that would alter respiratory mechanisms and the absence of severe obesity.

Results: Monotherapy with DMARD was 27 out of 60 patients (45\%) (Sulfasalazine $3 \mathrm{~g} /$ day) for a period of 1-48 months (mean value=19.4 (15.5) months) There were no differences in the angle of the Ct-Abd curve between patients with DMARD and DMARD-naive treatment $\left(38.2(14.5)^{\circ}\right.$ and $34.7(19.5)^{\circ}$ for sitting position, $49.3(18.1)^{\circ}$ and $47.2(23.1)^{\circ}$ in orthostatism, and $19.1(15.6)^{\circ}$ and 16.1 $(14.6)^{\circ}$ for clinostatism, $\left.\mathrm{p}<0.05\right)$. In the baseline study, the Ct-Abd patient angle was lower than the control group in sitting position $\left(36.3(17.3)^{\circ}\right.$ and $51.5(8.9)^{\circ}$ $\mathrm{p}=0.0002)$ in orthostatism $\left(48.1(20.8)^{\circ}\right.$ and $\left.62.4(12.5)^{\circ}, \mathrm{p}<0.01\right)$ or orthostatism $\left(17.4(15.0)^{\circ}\right.$ and $\left.24.5(9.8)^{\circ} \mathrm{p}<0.05\right)$. In the entire patient group, the Ct-Abd angle correlated negatively with BASFI in all three body positions $(r=-0.50, p<0.0001$ in the sitting position, $r=-0.36, p<0.01$ in orthostatism, $r=-0.47, p<0.0001$ in clinostatism); did not correlate with BASDAI, BASMI, or the modified Schoeber test in either of the three body positions.

In 15 AS patients who underwent repeated measurements of toracoabdominal movements while receiving their associated DMARD treatment (Methotrexate $15 \mathrm{mg} /$ week and Sulfasalazine $3 \mathrm{~g} /$ day) 3 months after treatment, the angle of the Ct-Abd slope was significantly higher than that of the fundamental study, in all bodily positions.

The Ct-Abd angle continued to increase, with increments less pronounced and reached significant value only between measurements of 3 months and 12 months. Improvements in standardised clinical signs following associated DMARD treatment followed a similar pattern, with scores at each interval significantly different from those measured in the baseline study, improvements continuing at a faster pace slowly after the third month.

In the control group, the angle of the slope of the Ct-Abd curve was not different in the two measurements in any of the body positions $\left(51.4(8.9)^{\circ}\right.$ and $50.7(9.3)^{\circ}$ in the sitting position, $62.4(12.4)^{\circ}$ and $61.6(11.8)^{\circ}$ in orthostatism, and $24.6(9.8)^{\circ}$ and $24.8(10.4)^{\circ}$ in clinostatism, $\left.\mathrm{p}<0.05\right)$. In orthostatism, the difference between the measurements was $0.8^{\circ}$ (confidence interval $95 \%-0.9$ to 2.52 , upper and lower boundaries of $6.6^{\circ}$ and $8.2^{\circ}$ ).

Conclusions: The slope of the $\mathrm{Ct}-\mathrm{Abd}$ curve during quiet breathing correlates negatively with BASFI and responds significantly to associated DMARD treatment and NSAIDs. Our data suggest that this measure can be targeted for further evaluation of its usefulness in monitoring chest involvement and its response to treatment in AS patients.

Disclosure of Interest: None declared

DOI: 10.1136/annrheumdis-2018-eular.3641

\section{AB0832 THE EFFECTS OF ANTI-TNF BIOLOGICAL AGENTS IN PATIENTS WITH SPONDYLOARTHRITIS: A COMPARATIVE STUDY}

F. Essafi, O. Saidane, I. Mahmoud, A. Ben Tekaya, R. Tekaya, L. Abdelmoula. rheumatology, Charles Nicolle Hospital, Tunis, Tunisia

Objectives: The aim of the present study was to compare the efficacy of three anti-TNF agents (adalimumab, infliximab and etanercept) in patients with spondyloarthritis $(\mathrm{SpA})$ at 24 weeks.

Methods: We achieved a retrospective descriptive and comparative monocentric study, on 49 patients, with SpA including ankylosing spondylitis (AS), psoriatic arthritis (PsA), enteropathic arthritis $(E A)$, reactive arthritis $(R e A)$ and undifferentiated spondyloarthritis (USpA) (according to Amor criteria, ASAS 2009 and CASPAR criteria), during 12 years (2004-2015). The patients were treated with at least one anti-TNF, during at least 6 months. Disease activity was assessed by the BASDAI, ASDAS, ESR and CRP. To compare mean differences between time points (week 0 versus week 24), a Wilcoxon test was applied. To compare efficacy between the 3 anti-TNF, a Mann-Whitney test was applied.

Results: Twenty three patients (47\%) had AS, 13 patients (27\%) had PsA and 11 patients $(22 \%)$ had EA. One patient had an uSpA, and 1 patient had a ReA. The mean age was 42,81 years $\pm 11,77$. The median age at disease onset was 29,41 years $\pm 11,29$. The mean disease duration was 10,16 years. Nineteen patients received etanercept (ETN), 18 infliximab (IFX) and 12 adalimumab (ADA). At six Months, the 3 anti-TNF showed improvement in the disease activity scores: BAS DAI $(p<0,0001)$, ASDAS CRP $(p<0,0001), \operatorname{ESR}(p<0,0001)$ and CRP $(p<0,0001)$ Sixty two percent of the patients have reached BASDAI 50 response at 6 months.

Abstract AB0832 - Table 1. Summary of median change from baseline to week 24 in clinical outcomes

\begin{tabular}{lccc}
\hline & Week 0 & Week 24 & $\mathrm{p}$ \\
\hline BASDAI & $6,04 \pm 2,28$ & $2,5 \pm 2,32$ & $<0,0001$ \\
ASDAS & $4,27 \pm 1,13$ & $2,17 \pm 1,3$ & $<0,0001$ \\
CRP & & & \\
CRP $(\mathrm{mg} / \mathrm{l})$ & 44,68 & 14,03 & $<0,0001$ \\
& $\pm 33,45$ & $\pm 10,09$ & \\
ESR $(\mathrm{mm})$ & 62,24 & 25,65 & $<0,0001$ \\
& $\pm 39,16$ & $\pm 24,97$ & \\
\hline
\end{tabular}

BASDAI: Bath Ankylosing Spondylitis Disease Activity Index, ASDAS: Ankylosing Spondylitis Disease Activity Score, C-Reactive protein, ESR: Erythrocyte Sedimentation Rate

At week 24, patients on ETN achieved more frequently a significant reduction of BASDAI ( $-5,13$ vs $-2,53 ; p=0,004)$, ESR $(-52,69$ vs $-16,64 ; p=0,006)$ and CRP $(-36,7$ vs $-21,34 ; p=0,009)$ than IFX. The efficacy comparison between ETN and ADA showed ETN superiority concerning BASDAI ( $-5,13$ vs $-2,04 ; p=0,004$ ) and ASDAS CRP $(-3,03$ vs $-1,4 ; p=0,006)$. The comparison ADA vs IFX had shown no significant efficacy differences.

Conclusions: Our results showed a BASDAI 50 response in $62 \%$ of patients. The comparison between the three biologics was consistent with a better efficacy of the ETN

Disclosure of Interest: None declared

DOI: 10.1136/annrheumdis-2018-eular.3362

\section{AB0833 REAL-WORLD EFFICACY AND SAFETY OF SECUKINUMAB: DATA FROM VERONA'S COHORT}

F. Martinis, C. Caimmi, A. Carletto, E. Fracassi, M. Rossini. Rheumatology, AOUI Verona, Verona, Italy

Background: Secukinumab has been approved for the treatment of active ankylosing spondilytis (AS) and psoriatic arthritis (PsA). Its efficacy has been demonstrated in phase III trials where eligibility criteria ensured a homogeneous population. Although this strategy reduces confounding factors, it does not guarantee the same results in the real world, where clinicians deal with advanced dis ease, comorbidities, adherence and persistence challenges.

Objectives: Aim of this study was to assess efficacy and safety of Secukinumab in real-world clinical practice.

Methods: Patients received Secukinumab (150 or $300 \mathrm{mg}$ ) at weeks 0,1,2,3 and 4 as induction therapy and then every 4 weeks as manteinance therapy. Assess ment of disease acrivity was done at months 0,6 and 12 using DAPSA, ASDAS BASDAI, BASFI, pain VAS

Results: 61 patients affected by PsA (65\% females, 35\% males) and 29 affected by AS (70\% males, 30\% females) were included. $64 \%$ of patients reached 12 months follow up. Baseline characteristics of both groups are shown in the tables below.

In the PsA cohort, the median DAPSA at baseline was 19.5 (IQR 9.6), at 6 months 9.09 (IQR 6.5, p<0.001), at 12 months 8.53 (IQR 6.9, $\mathrm{p}<0,001$ ). Median pain VAS showed a downward trend as well, from 6 at baseline (2 IQR) to 4.5 at 6 months (2 IQR) and 4 at 12 month (IQR 1.4). No differences emerged among PsA patterns. Clinical trials did not assess efficacy of Secukinumab in patients previously treated with biologic agents other than anti-TNF agents, due to exclusion criteria. We performed subgroup analysis to evaluate its efficacy in patients previously treated with Ustekinumab, finding a reduction in median DAPSA from 19.4 (IQR 9.8, $\mathrm{p}<0.001)$ at baseline to $8.7($ IQR $8.3, \mathrm{p}<0.001)$ at 1 year.

In the AS cohort, the median BASDAI at baseline was 5.5 (IQR 2.2), at 6 months 3.6 (IQR 1.2, $\mathrm{p}<0.001$ ), at 12 months 3.1 (IQR 1, $\mathrm{p}<0.001$ ). Median BASF decreased from 4.8 (IQR 2.7) to 3.8 (IQR 2.4, p<0.001) and 3 (IQR 2.4, p<0.001) at 6 and 12 months respectively. ASDAS recuced from 3.02 (IQR 1.65), to 1.21 (IQR 1.92, p<0.001) at 6 months and to 1.05 (IQR $0.7, p<0.001)$ at 1 years follow up. Median pain VAS dropped off from 7 at baseline (IQR 2$)$ to $4(I Q R, p<0.001)$ at 6 months and $3($ IQR, $p<0.001)$ at 12 months. Of note, patients with advanced dis ease (ankylosis of the spine) reported a decrease in pain and morning stiffness, thus improving their quality of life.

4 patients switched therapy at 6 months due to partial response, 1 patient experienced an expected adverse event (Candida infection). Overall, no serious side effects were observed and none resulted in Secukinimab discontinuation. 